\title{
All Semiconductor Low- $\Delta$ Photonic Crystal Waveguide for Semiconductor Optical Amplifier
}

\author{
Eiichi MIZUTA, Hideki WATANABE and Toshihiko BABA \\ Department of Electrical and Computer Engineering, Yokohama National University, 79-5 Tokiwadai, \\ Hodogaya-ku, Yokohama 240-8501, Japan
}

\begin{abstract}
An ultralow group velocity of light in a III-V semiconductor photonic crystal (PC) waveguide is expected to enhance the optical gain per unit length of the waveguide. We discuss the possibility of a compact semiconductor optical amplifier that utilizes a single-line-defect PC waveguide fabricated from an all semiconductor low- $\Delta$ slab with deep airholes. From photonic band and traveling-wave rate equation analysis, a gain of larger than $20 \mathrm{~dB}$ is expected in a short active PC waveguide of $10 \mu \mathrm{m}$ length, although the waveguide mode has a large radiation loss. We experimentally demonstrate such a waveguide in the passive wavelength regime. The waveguide is fabricated into a GaInAsP/InP wafer with high-mesa input/output waveguides. We show that the observed transmission characteristics of light correspond well to the photonic band theory.
\end{abstract}

Keywords photonic crystal, SOA, slow light, low group velocity, GaInAsP/InP

\section{Introduction}

In the research field of photonic crystals (PCs), the line defect waveguide is a major topic. Thus far, studies on this waveguide have been mostly devoted to their fabrication, fundamental characterizations, ${ }^{1-8)}$ and applications for passive devices such as filters ${ }^{9-11)}$ and delay lines. ${ }^{12-16)}$ The discussions on active device applications have been limited to those on microlasers ${ }^{17,18)}$ and standard-size stripe lasers. ${ }^{19)}$ However, this waveguide has an attribute that enhances the light-matter interaction near the photonic band edge, at which the group velocity of light $v_{\mathrm{g}}$ is markedly reduced. ${ }^{20,21)}$ Therefore, its applications for various active devices should be investigated further.

In this paper, we propose a compact semiconductor optical amplifier (SOA) or an optical gate device based on an active PC waveguide that is fabricated into a direct-transition-type semiconductor wafer. We expect an enhancement of the optical absorption and gain per unit length, a reduction in device size and power consumption, and a marked nonlinearity and cross-gain modulation among others because of the high optical density in the minute waveguide. In our previous experiments, we fabricated an airbridge GaInAsP PC slab, and performed the cw photopumping of the guide mode. ${ }^{22)}$ However, the observed optical gain was not obtained. The main reason for this was the marked heat generation caused by the very high thermal resistance of the order of $0.1 \mathrm{~K} / \mu \mathrm{W}$ in the PC slab, ${ }^{23)}$ which prevented the formation of a population inversion.

For this study, we considered the use of a PC waveguide consisting of a semiconductor core and cladding layers with deep airholes, because such waveguide allows a marked reduction in thermal resistance and the electrical control of the device. (Let us term this type of waveguide a low- $\Delta$ PC waveguide, where $\Delta$ is the relative refractive index difference between the core and cladding layers) Regarding such waveguides, many reports have discussed light propagation in wide channels such as three-line defects. ${ }^{3,11,13,19)}$ However, their photonic band is so complicated that we cannot expect a single-mode propagation in a wide spectral range. On the other hand, reports on single-line defect waveguides are limited because of the large radiation loss of the fundamental waveguide mode.,24) The attenuation of the guided mode is so serious that the light output is negligible in a sample of longer than $100 \mu \mathrm{m}$. In this study, we discuss a PC waveguide integrated with high-mesa rectangular channel waveguides for optical input and output (I/O), as shown Fig. 1, and similar to that reported in ref. 5). Since the propagation loss in the I/O waveguides is expected to be sufficiently low, we can evaluate the radiation loss in a passive PC waveguide having a moderately small length. Then, the next interesting question is whether it can be used as a SOA when the absolute value of the radiation loss is sufficiently low in a short PC waveguide, compared with the enhanced gain. If an active PC waveguide and passive I/O waveguides are monolithically integrated by, for example, epitaxial regrowth, the optical absorption in the I/O waveguides is eliminated and easy coupling with an 
external optical setup and a reduction in insertion loss are expected.

In $\S 2$, we first present a three-dimensional (3D) finite-difference time-domain (FDTD) analysis of the photonic band, group velocity $v_{g}$ and radiation loss of the waveguide mode in the low- $\Delta$ PC waveguide. In $\S 3$, we present the traveling-wave rate equation analysis of the SOA based on $v_{\mathrm{g}}$ and the radiation loss, and show that a large gain is expected even in a short waveguide of $9 \mu \mathrm{m}$ length. Finally, in $\S 4$, we describe the design of this waveguide integrated with $\mathrm{I} / \mathrm{O}$ waveguides and its fabrication into a GaInAsP/InP wafer with a simple passive core layer. We show the details of the light propagation characteristics and discuss their correspondence to the photonic band theory.

\section{Mode Analysis}

Regarding the waveguide mode in the low- $\Delta$ PC waveguide, a large radiation loss is theoretically discussed in ref. 25). However, the loss characteristics, modal profiles, and dependence on structural parameters are not fully discussed. In addition, the group index is not quantitatively shown, although it is important for various applications. We calculated the photonic band, loss and group index characteristics by completely modeling the structure, which was experimentally fabricated. Here, the plane-wave expansion method cannot be used because of the radiation loss. We employ 3D FDTD analysis, in which the periodic boundary condition is applied to the direction of light propagation and the absorbing boundary condition is applied to the other directions. The unit-cell model of the waveguide is shown in Fig. 2(a). The index and thickness $t$ of the GaInAsP core layer are 3.45 and $1.32 a$, respectively, where $a$ is the lattice constant. The index of the InP cladding layers is 3.17 , and the thickness of the upper cladding layer $d$ is $1.58 a$. Above the upper cladding layer is air, which is assumed to have a refractive index of 1.0. The diameter of each airhole $2 r$ is $0.632 a$. The depth of each airhole $h$ from the top of the upper cladding layer is set at 5.26a so that the holes perforate the core layer. The single line defect centered in the model is sandwiched by five triangular lattice airholes on each side. The initial wave excitation is given for the transverse electric field at the core center (TE polarization).

Figure 2(b) shows the calculated photonic band diagram. Similarly to that in a standard high- $\Delta$ PC slab, ${ }^{2)}$ a low-frequency slab-edge mode and two waveguide modes appear. Here, the waveguide modes are essentially leaky modes with out-of-plane radiation loss due to the low $\Delta$. In this study, we focus on the lower-order mode as a target waveguide mode. If we consider the radiation loss to be on the acceptable level, the normalized frequency $\omega a / 2 \pi c$ of this mode ranges from 0.246 to above 0.3 , because there are no critical restrictions such as light line. Figure 2(a) also shows the cross-sectional distribution of the transverse electric field of the mode when the wavenumber $k$ in the direction of light propagation is $0.475(2 \pi / a)$. A symmetric mode profile with an antinode at the core center is observed. The three-antinode profile in the core region changes to one-antinode profile, depending on the position of the cross-section along the waveguide, as is usually observed in a high- $\Delta$ PC slab waveguide.

Figure 3 shows the group index $n_{\mathrm{g}}$ evaluated from the slope of the band curve and the loss evaluated from the lifetime of the mode. The group index $n_{\mathrm{g}}$ is $4-5$ when the frequency is far from the band edge, but becomes higher than 400 near the band edge of $\omega a / 2 \pi c=0.246$. Thus, an ultralow group velocity is expected even in the low- $\Delta$ PC waveguide. Similarly to that in the high- $\Delta$ PC waveguide, the frequency bandwidth, which gives the ultralow group velocity, is very narrow. However, the bandwidth can be moderately expanded using a chirped structure ${ }^{12,15)}$ or by optimizing the waveguide parameters. ${ }^{26,27)}$ These are applicable to PC SOAs. On the other hand, the loss increases as $n_{\mathrm{g}}$ increases. The loss is $3 \times 10^{-3}-8 \times 10^{-3} n_{\mathrm{g}} \mathrm{dB} / a$ at $\omega a / 2 \pi c$ far from the band edge, and $1 \times 10^{-3}$ $2 \times 10^{-3} n_{\mathrm{g}} \mathrm{dB} / a$ near the band edge. For $a=0.38 \mu \mathrm{m}$, for example, the loss becomes $7.5 \times 10^{2} \mathrm{~dB} / \mathrm{cm}$ at $n_{\mathrm{g}}=4$, and $6.2 \times 10^{3} \mathrm{~dB} / \mathrm{cm}\left(=1.4 \times 10^{3} \mathrm{~cm}^{-1}\right)$ at $n_{\mathrm{g}}=100$. Although the loss near the band edge is very large for a passive waveguide, it will not be crucial for the SOA because the device can be markedly shortened owing to the large $n_{\mathrm{g}}$ and the enhanced optical gain, as shown below. Note that the loss coefficient excluding $n_{\mathrm{g}}$ decreases toward the band edge. This means that the extent of increase in loss caused by the group index effect is lower than that of the increase in gain.

We also examined the dependence of the loss on some structural parameters to determine the influence of fabrication errors. For example, the increase in loss is limited to less than $6 \%$ for a $10 \%$ 
change in either core thickness $t$ or waveguide width $W$. When the thickness of the upper cladding $d$ changes by 20 or $40 \%$, the loss is 1.6 or 3.4 times larger, respectively. The most sensitive parameter is the airhole depth $h$; if it were reduced by 10,20 , or $30 \%$, the loss would be $1.3,3.5$, and 10 times larger. The thicknesses of the core and cladding layers can be controlled by the epitaxy of the wafer used. Waveguide width can be determined by lithography. Therefore, the most important issue is to form airholes with sufficient depths.

\section{Rate Equation Analysis}

To confirm the operation of the PC SOA, we analyzed the gain characteristics using traveling-wave rate equations. For the carrier density $N\left(\mathrm{~cm}^{-3}\right)$, as a function of position $z$ and time $t$, and the photon density $S\left(\mathrm{~cm}^{-3}\right)$ of the waveguide mode, as a function of $t$, the rate equations are ${ }^{28)}$

$$
\begin{aligned}
& \frac{d N}{d t}=P-\frac{N}{\tau_{\mathrm{c}}}-\Gamma g(N) S, \\
& \frac{n_{\mathrm{g}}}{c} \frac{d S}{d t}+\frac{d S}{d z}=\frac{n_{\mathrm{g}}}{c}\left(\Gamma g(N)-\frac{c}{n_{\mathrm{g}}} \alpha\right) S,
\end{aligned}
$$

where $P\left(\mathrm{~cm}^{-3} \mathrm{~s}^{-1}\right)$ is the pump rate density, $\tau_{\mathrm{c}}(\mathrm{s})$ is the carrier lifetime, $\Gamma$ is the optical confinement factor, $g(N)\left(\mathrm{s}^{-1}\right)$ is the gain coefficient, $c$ is the light velocity in vacuum, $\alpha\left(\mathrm{cm}^{-1}\right)$ is the radiation loss calculated by FDTD analysis, and $\tau_{\mathrm{sp}}(\mathrm{s})$ is the spontaneous emission lifetime. The spontaneous emission coupled to the waveguide mode and the facet reflection are ignored. In addition, we assume

$$
\begin{aligned}
& \frac{1}{\tau_{\mathrm{c}}}=A+B N+C N^{2}, \\
& g(N)=\frac{C}{n_{\mathrm{eq}}} G_{0}\left(N-N_{0}\right), \\
& \frac{1}{\tau_{\mathrm{sp}}}=B N,
\end{aligned}
$$

where $A\left(\mathrm{~s}^{-1}\right)$ is the surface recombination rate, $B\left(\mathrm{~cm}^{3} \mathrm{~s}^{-1}\right)$ is the radiative recombination coefficient, $C$ $\left(\mathrm{cm}^{6} \mathrm{~s}^{-1}\right)$ is the Auger recombination coefficient, $G_{0}\left(\mathrm{~cm}^{2}\right)$ is the linear gain coefficient, and $N_{0}\left(\mathrm{~cm}^{-3}\right)$ is the transparency carrier density. In actual calculations, a $1.55-\mu \mathrm{m}-\mathrm{GaInAsP}$ bulk active layer with width $W$, thickness $t$, and length $L$ is assumed. The parameters used are summarized in Table I.

Figure 4 compares the graphs of the gain $G=\left(n_{\mathrm{g}} / c\right) g$ versus the output power characteristics of a standard bulk SOA and the PC SOA. Here, important differences between these two SOAs are the group index, device length and radiation loss, i.e., $n_{\mathrm{g}}=3, L=300 \mu \mathrm{m}$, and $\alpha=30 \mathrm{~cm}^{-1}$ for the standard SOA, while $n_{\mathrm{g}}=100, L=9 \mu \mathrm{m}$, and $\alpha=1427 \mathrm{~cm}^{-1}$ for $a=0.325 \mu \mathrm{m}$ for the PC SOA, respectively. The injection current $I$ is obtained from the relation $P=I / e W t L$ ( $e$ is the electron charge). As observed in Fig. 4, $G=25 \mathrm{~dB}$ is expected to occur at $I>50 \mathrm{~mA}$ in the standard SOA, while $G=25$ $\mathrm{dB}$ occurs at $I=3 \mathrm{~mA}$ for the PC SOA. This difference arises from differences in $n_{\mathrm{g}}$ and $L$. However, the large radiation loss can be compensated by the large gain, and a large net gain is expected in a very short device. On the other hand, the 3-dB saturated output power $P_{3 \mathrm{~dB}}$ is low: e.g., $P_{3 \mathrm{~dB}}=-5 \mathrm{dBm}$ at $I$ $=3 \mathrm{~mA}$ for the PC SOA. Such a low saturated power is essential because the output power is generally restricted by injection current. Therefore, the PC SOA is not suitable as a booster amplifier with high output power but is suitable as a preamplifier and an optical gate and for compensation of local loss with an output power up to from -10 to $0 \mathrm{dBm}$. The PC SOA is still very effective and attractive for constructing functional active photonic circuits with PCs.

\section{Experiment}

The device shown in Figs. 1 and 2 was fabricated to confirm the passive characteristics of the low- $\Delta$ PC waveguide. The wafer used consisted of an InP top cladding layer of $0.73 \mu \mathrm{m}$ thickness $(d / a$ $=2.25$ to 1.95 for $a=0.325$ to $0.375 \mu \mathrm{m}$ ), a $1.32-\mu \mathrm{m}$-GaInAsP bulk core layer of $0.45 \mu \mathrm{m}$ thickness ( $t / a=1.38$ to 1.20 ) and an InP substrate. The parameters of the PC were set at $a=0.325,0.350$, or 
$0.375 \mu \mathrm{m}$ and $2 r=0.2 \mu \mathrm{m}(2 r / a=0.53$ to 0.62$)$. In addition, the channel width $W$ was changed by -50 , 0 , and $50 \mathrm{~nm}(\Delta W / a=-0.14$ to 0.14 for $a=0.325 \mu \mathrm{m})$ from the standard width of the single line defect. The length of the PC waveguide $L$ was elongated to $15 \mu \mathrm{m}$ to be able to clearly observe the loss and related effects of the PC waveguide. The I/O waveguides were designed to be $0.5 \mu \mathrm{m}$ in width at the portions connecting to the PC waveguide. The waveguides were expanded to $2.0 \mu \mathrm{m}$ width through adiabatic tapers of $50 \mu \mathrm{m}$ length so that the coupling efficiency with an external optical setup was improved. The total length of the device including the $\mathrm{I} / \mathrm{O}$ waveguides was nearly $300 \mu \mathrm{m}$, which was determined by the cleavage.

For the pattern generation of the device, an $e$-beam writer (ELS7300, Elionix) was used with a positive resist (ZEP520A, Zeon) of $0.3 \mu \mathrm{m}$ thickness. Because the minimum grid size of the $e$-beam writer was as large as $25 \mathrm{~nm}$ for a chip size of $500 \times 500 \mu \mathrm{m}^{2}$ and the proximity effect was marked, fine exposure adjustments were carried out for each pattern. The patterns of the I/O waveguides were expanded to $0.7 \mu \mathrm{m}$ at the connecting portions. For the formation of deep airholes, we first attempted to use $\mathrm{Cl}_{2}$-based inductive coupled plasma (ICP) etching with a metal mask. However, it was difficult to achieve $2 r=0.2 \mu \mathrm{m}$ and $h>1.5 \mu \mathrm{m}$ for $a<0.4 \mu \mathrm{m}$, which were required to realize the calculation model at $\lambda=1.55 \mu \mathrm{m}$. We finally employed HI/Xe ICP etching. ${ }^{29)}$ Because substrate heating is not necessary in HI/Xe ICP etching, the resist can be directly used as an etching mask. As for the etching of InP, the details of the optimum conditions for the etching machine (RIE-200ip, Samco) are shown in ref. 29). To obtain similar etching conditions for GaInAsP, we slightly modified HI and Xe flow rates to 0.2 and $0.1 \mathrm{sccm}$, respectively, the gas pressure to $0.28 \mathrm{~Pa}$, the substrate temperature to $70{ }^{\circ} \mathrm{C}$, the ICP power to $100 \mathrm{~W}$, and the bias power to $200 \mathrm{~W}$. Under these conditions, the etching rates of InP and 1.32- $\mu \mathrm{m}$-GaInAsP were 80 and $50 \mathrm{~nm} / \mathrm{min}$, respectively. Figure 5 shows the top and cross-sectional views of the fabricated device. The depths of the airholes and high-mesa waveguides were all $2.0 \mu \mathrm{m}$ for an etching time of $32 \mathrm{~min}$. Owing to the physical etching of ions, the bottoms of the airholes are slightly sharpened. The sidewall angle in the core layer is almost $90^{\circ}$ to the substrate plane.

For the measurements, a TE-polarized tunable laser light was coupled to the cleaved end facet of the input waveguide using two objective lenses. The light output from the output waveguide was observed using a vidicon camera and simultaneously measured using an optical power meter. Figure 6 shows the measured transmission spectra, where the intensity is normalized to that when no sample is present so that the total insertion loss can be evaluated. The large drop in light transmission for $\lambda<$ $1.32 \mu \mathrm{m}$ is caused by the interband absorption by the core layer. Light transmission was observed on the longer-wavelength side of the electronic band edge. Fine fringes in the spectra were caused by the Fabry-Perot resonance between the input and output ends of the I/O waveguides. Curve A shows the results for the sample, for which the PC waveguide was replaced by the high-mesa waveguide of 0.5 $\mu \mathrm{m}$ width. The insertion loss for this case was $12 \mathrm{~dB}$, which is sensitive to the ICP etching conditions and the corresponding sidewall roughness; it was reduced to $\sim 5 \mathrm{~dB}$ for the best case. Therefore, the coupling loss from the setup to the waveguide is estimated to be less than $2.5 \mathrm{~dB}$ per port. Curves $\mathrm{B}, \mathrm{C}$, and D in Fig. 6(a) are spectra obtained at different lattice constants $a$, where $\Delta W$ was fixed at $-50 \mathrm{~nm}$. The curves in Fig. 6(b) are spectra obtained at different $\Delta W$ values, where $a$ is fixed at $0.325 \mu \mathrm{m}$. As shown here, the cutoff wavelength on the longer-wavelength side shifts with $a$ and $\Delta W$. The arrows in the figure indicate the band edges of the waveguide mode estimated from the photonic band. They coincide well with the cutoff wavelengths of the experimental transmission spectra. Thus, we can conclude that the spectra are dominated by the photonic band characteristics. From the comparison of the highest transmission intensity between curves $\mathrm{A}$ and $\mathrm{B}$, the sum of the PC waveguide loss and the connection loss between the PC waveguide and the I/O waveguides is estimated to be less than $3 \mathrm{~dB}$. From the results shown in Fig. 3, the discrepancy in $d$ between the calculation model and the experiment, and the length of the PC waveguide $(L=15 \mu \mathrm{m})$, the PC waveguide loss at a wavelength far from the band edge is estimated to be less than $\sim 1.6 \mathrm{~dB}$. Therefore, the connection loss between the PC waveguide and the $\mathrm{I} / \mathrm{O}$ waveguides is estimated to be less than $1 \mathrm{~dB}$ per port. 


\section{Conclusions}

We discussed the possibility of a PC SOA, in which the ultralow group velocity of the photonic band enhances optical gain. We proposed the use of a single-line-defect PC waveguide with an all-semiconductor low- $\Delta$ slab as an active region and high-mesa waveguides as passive $\mathrm{I} / \mathrm{O}$ waveguides for the PC SOA. In the photonic band calculation, a group index up to 400 was estimated with a large radiation loss of the order of $10^{3} \mathrm{~dB} / \mathrm{cm}$ near the photonic band edge. It was also shown by traveling-wave rate equation analysis that, owing to the low group velocity, a $>25 \mathrm{~dB}$ gain is expected in a short active waveguide of $9 \mu \mathrm{m}$ length, even with the large radiation loss. In the experiment, such a PC waveguide was fabricated with I/O waveguides into a GaInAsP/InP passive wafer, and the clear light transmission was observed in the wavelength range from 1.3 to $1.6 \mu \mathrm{m}$. The observed dependence on structural parameters corresponded well to the photonic band calculation.

For realizing a PC SOA, active and passive integrations are necessary. Here, the PC waveguide was formed in the active region with a narrow electronic band gap and the I/O waveguides were formed in the passive region with a wide electronic band gap. Such integrations can be realized by epitaxial regrowth, which is reported elsewhere. ${ }^{30)}$

\section{Acknowledgments}

This work was partly supported by a Grant-in-Aid from the Japan Society for the Promotion of Science, and a Grant-in-Aid, the IT Program and 21st Century COE Program of the Ministry of Education, Culture, Sports, Science and Technology.

\section{References}

1) T. Baba, N. Fukaya and J. Yonekura: Electorn. Lett. 35 (1999) 654.

2) T. Baba, A. Motegi, T. Iwai, N. Fukaya, Y. Watanabe and A. Sakai: IEEE J. Quantum Electron. 38 (2002) 743.

3) C. J. M. Smith, H. Benisty, S. Olivier, M. Rattier, C. Weisbuch, T. F. Krauss, R. M. De La Rue, R. Houdré and U. Oesterle: Appl. Phys. Lett. 77 (2000) 2813.

4) M. Notomi, A. Shinya, K. Yamada, J. Takahashi, C. Takahashi and I. Yokohama: Electron. Lett. 37 (2001) 293.

5) T. J. Karle, D. H. Brown, R. Wilson, M. Steer and T. F. Krauss: IEEE J. Sel. Top. Quantum Electron. 8 (2002) 909

6) W. Bogaerts, V. Wiaux, D. Taillaert, S. Beckx, B. Luyssaert, P. Bienstman and R. Baets: IEEE J. Sel. Top. Quantum Electron. 8 (2002) 928.

7) S. J. McNab, N. Moll and Y. A. Vlasov: Opt. Express 11 (2003) 2927.

8) Y. Sugimoto, Y. Tanaka, N. Ikeda, Y. Nakamura, K. Asakawa and K. Inoue: Opt. Express 12 (2004) 1090.

9) M. Notomi, A. Shinya, S. Mitsugi, E. Kuramochi and H.-Y. Ryu: Opt. Express. 12 (2004) 1551.

10) H. Takano, B-S. Song, T. Asano and S. Noda: Appl. Phys. Lett. 86 (2005) 241101.

11) E. Schwoob, H. Benisty, C. Weisbuch, C. Cuisin, E. Derouin, O. Drisse, G. H. Duan, L. Legouezigou, O. Legouezigou and F. Pommereau: Opt. Express 12 (2004) 1569.

12) D. Mori and T. Baba: Appl. Phys. Lett. 85 (2004) 1101.

13) H. Gersen, T. J. Karle, R. J. P. Engelen, W. Bogaerts, J. P. Korterik, N. F. van Hulst, T. F. Krauss and L. Kuipers: Phys. Rev. Lett. 94 (2005) 073903.

14) M. L. Povinelli, S. G. Johnson and J. D. Joannopoulos: Opt. Express 13 (2005) 7145.

15) D. Mori and T. Baba: Opt. Express 13 (2005) 9398.

16) Y. A. Vlasov, M. O'Boyle, H. F. Hamann and S. J. McNab: Nature 438 (2005) 65.

17) A. Sugitatsu and S. Noda: Electron. Lett. 39 (2003) 213

18) K. Inoshita and T. Baba: Electron. Lett. 39 (2003) 844.

19) S. Mahnkopf, R. März, M. Kamp, G-H. Duan, F. Lelarge and A. Forchel: IEEE Photonics Technol. Lett. 40 (2004) 1306.

20) M. Notomi, K. Yamada, A. Shinya, J. Takahashi, C. Takahashi and I. Yokohama: Phys. Rev. Lett. 87 (2001) 253902

21) K. Kiyota, T. Kise, N. Yokouchi, T. Ide and T. Baba: to be published in Appl. Phys. Lett. 89 (2006).

22) T. Baba, E. Mizuta, M. Shiga and K. Inoshita, Int. Symp. Quantum Dots and Photonic Crystals, 2003, P-33.

23) K. Inoshita and T. Baba: IEEE J. Sel. Top. Quantum Electron. 9 (2003) 1347.

24) A. Talneau, M. Mulot, S. Anand, S. Olivier, M. Agio, M. Kafesaki and C. M. Soukoulis: Photonic 
Nanostruct. Fundam. Appl. 2 (2004) 1.

25) W. Kuang, C. Kim, A. Stapleton, W. J. Kim and J. D. O'Brien: Opt. Lett. 28 (2003) 1781.

26) A. Sakai, I. Katoh, D. Mori, T. Baba and Y. Takiguchi: Proc. IEEE/LEOS Annual Meet., 2004, ThQ5.

27) A. Yu. Petrov and M. Eich: Appl. Phys. Lett. 85 (2004) 4866.

28) T. Saitoh and T. Mukai: IEEE J. Quantum Electron. 23 (1987) 1010.

29) T. Ide, J. Hashimoto, K. Nozaki, E. Mizuta and T. Baba: Jpn. J. Appl. Phys. 45 (2006) L102.

30) E. Mizuta, T. Ide, J. Hashimoto, K. Nozaki, T. Baba T. Kise, K. Kiyota and N. Yokouchi: Pacific Rim Conf. Laser and Electro-Optics, 2005, CThE1-4.

31) Table I. Calculation parameters.

\begin{tabular}{lll}
\hline Symbol & Parameter & Value \\
\hline$n_{\mathrm{eq}}$ & Equivalent modal index of standard SOA & 3.0 \\
$\Gamma$ & Optical confinement factor & 0.191 \\
$G_{0}$ & Linear gain efficient $\left(\mathrm{cm}^{2}\right)$ & $4 \times 10^{-16}$ \\
$N_{0}$ & Transparent carrier density $\left(\mathrm{cm}^{-3}\right)$ & $2.0 \times 10^{18}$ \\
$A$ & Surface recombination rate $\left(\mathrm{s}^{-1}\right)$ & $2.0 \times 10^{8}$ \\
$B$ & Spontaneous recombination rate $\left(\mathrm{cm} \cdot \mathrm{s}^{-1}\right)$ & $1.0 \times 10^{-10}$ \\
$C$ & Auger recombination rate $\left(\mathrm{cm}^{6} \cdot \mathrm{s}^{-1}\right)$ & $5.0 \times 10^{-29}$ \\
$\beta$ & Spontaneous emission factor & 0 \\
\hline
\end{tabular}

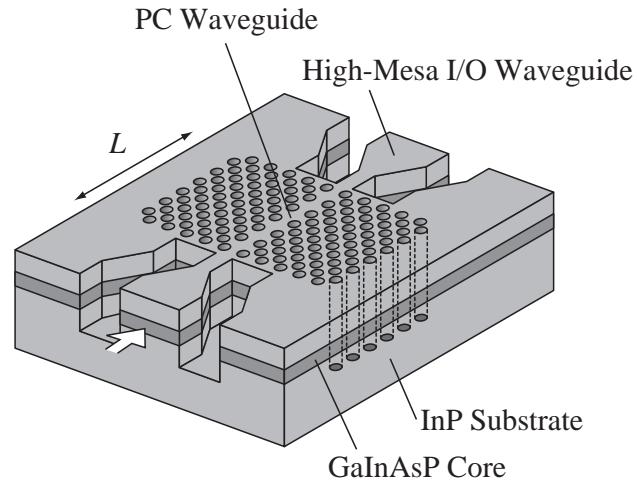

Fig. 1. Schematic of studied device. The low- $\Delta$ PC waveguide is integrated with high mesa $\mathrm{I} / \mathrm{O}$ waveguides on InP-based wafer. When the core layer of the PC waveguide is changed to an active layer with a narrower electronic bandgap by e.g. epitaxial regrowth, it acts as an SOA or a gate device.

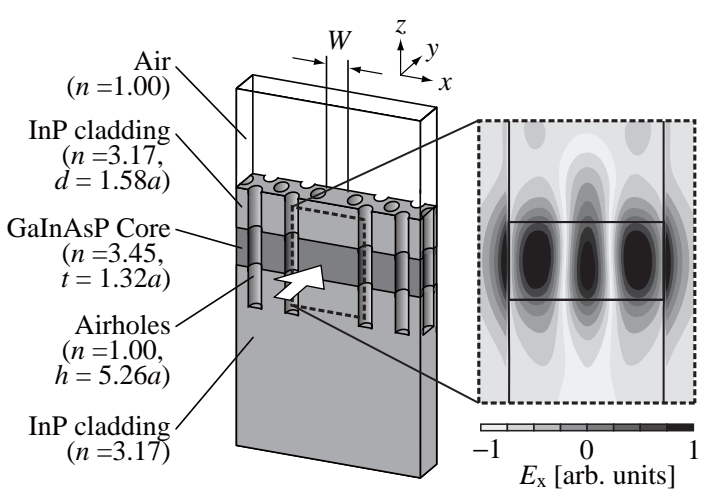

(a)

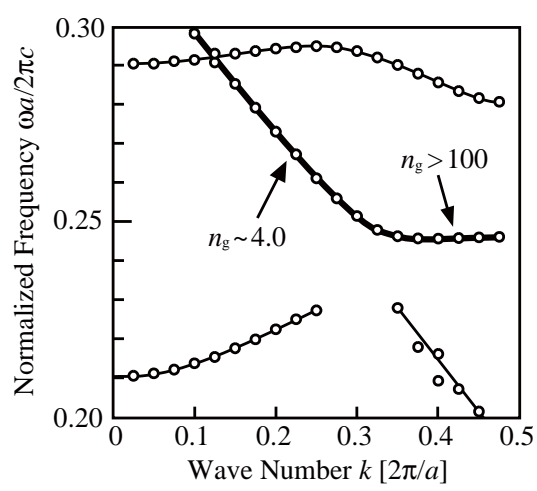

(b)

Fig. 2. 3D FDTD analysis of low- $\Delta$ PC waveguide. (a) Calculation model and electronic field distribution of lower-order waveguide mode. (b) Photonic band diagram. 


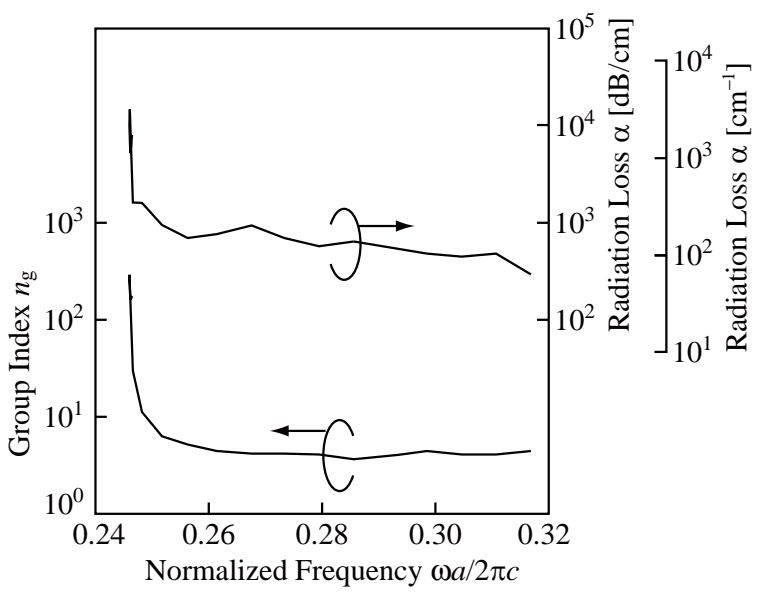

Fig. 3. Group index and radiation loss characteristics of lower-order waveguide mode in low- $\Delta$ PC waveguide.

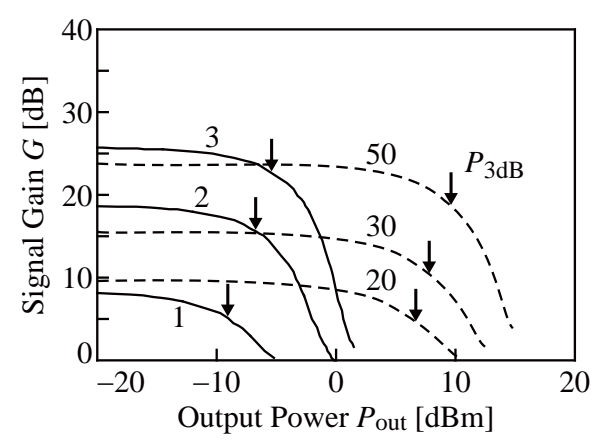

Fig. 4. Gain versus output power characteristics expected for PC SOA (solid lines) and conventional SOA (dashed lines).

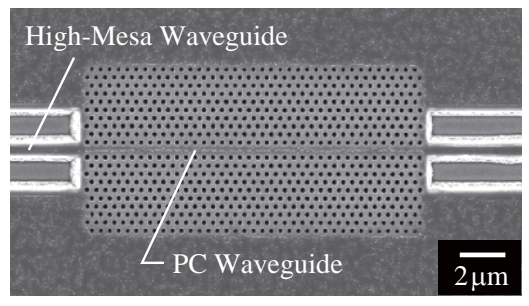

(a)

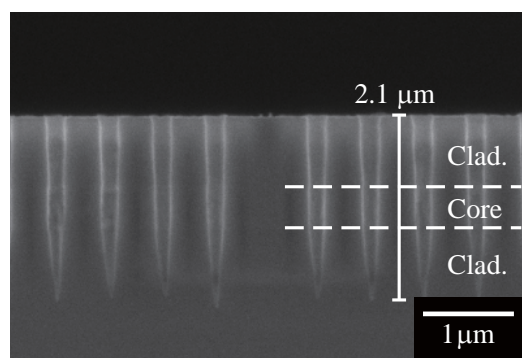

(b)

Fig. 5. Scanning electron micrographs of fabricated device. (a) Top view. (b) Cross-sectional view.

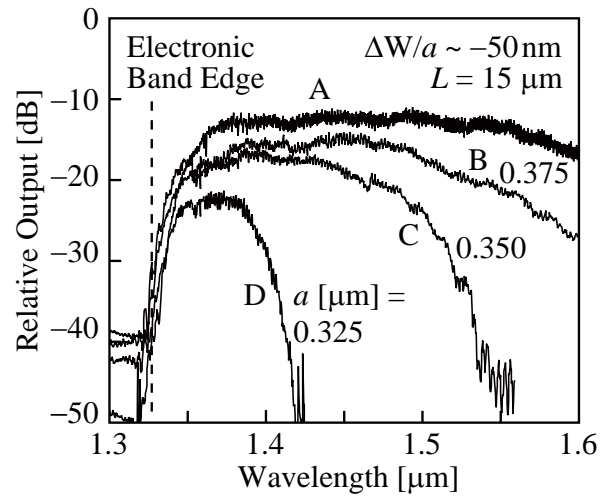

(a)

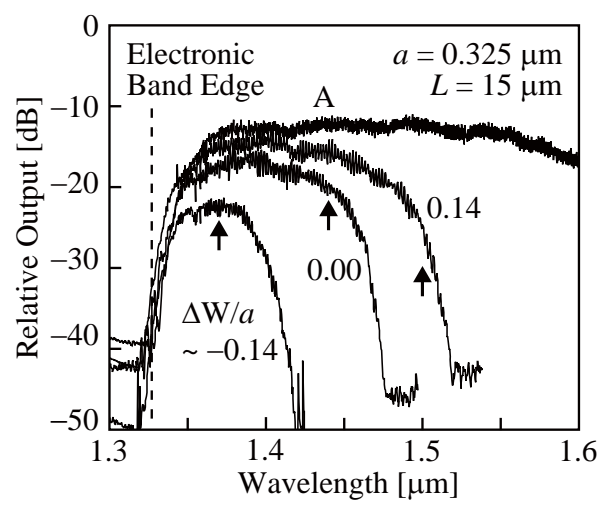

(b)

Fig. 6. Light transmission spectra of PC waveguides. (a) Comparison of spectra at different lattice constants. (b) Comparison of spectra at different waveguide widths. The arrows indicate band edges calculated by FDTD. 